\title{
O papel do FMEA no processo de tomada de decisão em desenvolvimento de novos produtos: Estudo em uma Empresa Automotiva
}

\author{
Paulo Augusto Cauchick Miguel, Dr. \\ André Segismundo, Mestrando \\ Departamento de Engenharia de Produção, Escola Politécnica da USP, São Paulo/SP
}

Atualmente, um efetivo gerenciamento de riscos tornou-se um elemento essencial para o sucesso em gerenciamento de projetos. Uma gestão de riscos não eficaz pode levar o projeto a atrasos, falhas e, muitas vezes até ao cancelamento. É neste contexto que o presente trabalho apresenta uma análise do papel do FMEA no processo de tomada de decisão em desenvolvimento de produtos de uma montadora de veículos. Como resultados parciais, observou-se um avanço na sistematização e disponibilidade de algumas informações antes não disponíveis para o processo decisório e, durante a fase inicial do projeto piloto, uma redução da necessidade de loopings de projeto e planejamento de testes e do número de protótipos necessários para a aprovação dos componentes. Como conclusões principais, tem-se uma possível influência positiva no processo de tomada de decisão do desenvolvimento de produtos, inferida através de uma melhor alocação dos recursos utilizados até o momento para o projeto piloto.

Palavras-chave: Gerenciamento de riscos, FMEA, Tomada de decisão.

Today, effectively managing risk is an essential element of successful project management. A no effective risk management could take project to delay failures or even to cancellation. At this context the current work presents an analysis of the FMEA rule at the product development decision making process at an automotive assembly company. As partial results, it was observed a progress at some no before available information's systematization and availability for the decision making process and, during the initial phase of the pilot project, a reduction at the need of project and test planning loopings and at the necessary prototypes number for the components approval. As principal conclusions, there is the possible positive influence at the product development decision making process, observed through a better resources allocation for the pilot project until the moment.

Keywords: Risk Management, FMEA, Decision making.

\section{Introdução}

Em tempos de competição e globalização cada vez maiores, o sucesso dos projetos de uma organização se tornou ainda mais decisivo para a performance do negócio da mesma e, ainda assim, vários projetos sofrem atrasos, mudanças de escopo, falhas e, no limite podem até ser cancelados (SHENHAR et al 2002). Em geral, muitos desses problemas podem ser gerados por uma gestão não eficaz dos riscos em projetos, a qual tem se tornado cada vez mais fundamental para o sucesso no gerenciamento dos mesmos (CARBONE; TIPPET, 2004). No entanto, técnicas e ferramentas de Gerenciamento de Riscos, as quais são desenvolvidas e utilizadas para aumentar as chances de sucesso de um projeto, são ainda muito pouco difundidas e empregadas (KUMAR, 2002).

Os riscos em projetos podem ser definidos como eventos indesejáveis que podem causar atrasos, gastos excessivos, resultados insatisfatórios para o projeto, organização, sociedade, meio ambiente e 
até, fracasso total do mesmo (SHENHAR et al., 2002). De acordo com o PMBOK (PMI, 2004), um risco de projeto é um evento ou condição incerta que, se ocorrer, terá um efeito positivo ou negativo sobre pelo menos um objetivo do projeto, como prazo, custo, escopo ou qualidade. Um risco pode ter mais de uma causa e, caso ocorra, pode ter mais de um impacto em mais de uma dimensão do projeto. De acordo com Carvalho e Rabechini. (2005), os riscos podem ainda ser identificados e agrupados em categorias, que devem refletir fontes comuns de risco para o projeto. Estas categorias são: riscos técnicos, riscos da gestão do projeto, riscos da organização e riscos externos (PMI, 2004). Os riscos técnicos, os quais constituem o foco de análise do presente trabalho, envolvem aqueles oriundos do uso de tecnologia não provada ou complexa, da exigência de atingir metas de desempenho não realistas, ou de mudança na tecnologia usada ou nas normas industriais durante o projeto. A categoria de riscos da gestão de projeto é caracterizada pelos riscos derivados da alocação inadequada de recursos, estimativas irrealistas e uma qualidade inadequada do plano de projeto. Para os riscos organizacionais, são os objetivos do projeto (custo, prazos, escopo) que podem estar incoerentes, falta de priorização de projetos e o financiamento inadequado ou interrompido. Por fim, os riscos externos são aqueles causados por alterações em legislações, mudanças nas tendências de mercado, questões trabalhistas e alteração nas prioridades dos patrocinadores do projeto (CARVALHO; RABECHINI, 2005).

Apesar das definições de risco de projeto serem claras e concisas e a determinação de possíveis riscos técnicos em sistemas bem conhecidos e sobre os quais se possui uma ampla base de informações sobre seus modos de falha serem relativamente simples (PATÉ-CORNELL, 2002), o gerenciamento dos riscos ao longo do ciclo de vida de um projeto ainda não se mostra maduro. Em seu estudo, Shenhar et al. (2002) concluiu que o gerenciamento de riscos está ainda em sua infância e que, em função disso, maiores esforços são necessários quanto à aplicação, treinamento, desenvolvimento de ferramentas e pesquisas quanto ao assunto.

Shenhar (2001) conclui ainda em outro estudo, que metodologias de gerenciamento de projetos e riscos não podem ser padronizadas para todos os tipos de projetos, e sim, devem ser adaptadas quanto a sua natureza de escopo e incerteza metodológica. Alguns estudos sugerem modelos (frameworks) específicos para gerenciamento de riscos em projetos de tecnologia da informação (KUMAR, 2002; MARTINEZ, 2004), em projetos de distribuição de energia elétrica (PEROBELLI, 2004) e, em alguns casos, para identificação dos riscos técnicos em desenvolvimento de novos produtos (KAPLAN et al., 2001; PUENTE et al., 2002; TRAMMELL, et al., 2004; CARBONE et al., 2004). Estes últimos são geralmente baseados no conceito de análise qualitativa de riscos gerada pela utilização do método FMEA (Failure Modes and Effects Analysis), o qual é um dos mais difundidos entre as empresas com o intuito de colaborar na determinação de prioridades no processo de gerenciamento de riscos técnicos através de pontos fracos dos projetos e conseqüentemente otimizando a alocação de recursos de testes e de engenharia (PATÉ-CORNELL, 2002; MADDOX, 2005).

Considerando-se ainda que o planejamento e gerenciamento do projeto de desenvolvimento de um novo produto, ou portfolio de produtos, pode ser classificado em termos de nível de decisão em problemas como um problema não estruturado, ou seja, aquele de médio a alto grau de incerteza e complexidade, onde tanto os possíveis cenários quanto os critérios de decisão não estão fixados $a$ priori (SHIMIZU, 2006), se faz de suma importância a gestão de riscos para auxiliar no seu processo decisório. De acordo com Carvalho et al. (2005), para tomar as decisões de forma adequada neste ambiente deve-se estar atento às singularidades de cada organização, como grau de aceitação ao risco e padrões corporativos para o planejamento e gestão dos riscos.

É neste contexto que o presente trabalho apresenta por objetivo geral analisar de forma qualitativa o papel do FMEA no processo de tomada de decisão em desenvolvimento de novos produtos de uma empresa automotiva. A seção 2 apresenta conceitos de análise de risco e FMEA, a seção 3 os métodos utilizados na pesquisa, as seções 4 e 5 ilustram respectivamente o conceito de riscos técnicos com FMEA, o processo de tomada de decisão bom base nos riscos e seus primeiros resultados, utilizados 
pela empresa objeto de estudo. A seção 6 apresenta outros resultados iniciais e relevantes do trabalho e por fim as conclusões são apresentadas na seção 7 seguida das referências bibliográficas.

\section{FMEA e análise de risco - conceitos}

O método FMEA surgiu em 1949 na Indústria Militar Americana e nos anos 60 a NASA, através do Apollo Space Program, foi a pioneira no seu desenvolvimento e evolução. O FMEA consiste em uma técnica de análise que foi desenvolvida para ser aplicada principalmente a componentes (hardware), cujo objetivo primordial é "radiografar" cada um dos componentes de um sistema a fim de levantar todas as maneiras pelas quais o componente possa vir a falhar e avaliar quais os efeitos que estas falhas acarretam sobre os demais componentes e sobre o sistema (instalação, equipamento, etc.) (CARBONE; TIPPET, 2004; SIMÕES, 2004). É um método útil para documentar de forma organizada os modos e os efeitos de falhas de componentes, ou seja, investiga-se o componente a fim de levantar todos os elementos, incluindo as ações inadequadas do ser humano, que possam interromper ou degradar o seu funcionamento e/ou do sistema ao qual o componente pertença (SIMÕES, 2004). Assim, de acordo com Maddox (2005), o FMEA constitui uma das técnicas de análise de risco mais utilizadas no domínio de engenharia de produto, a qual analisa os possíveis modos de falhas dos componentes de um sistema e indica os efeitos e a criticidade destas falhas sobre outros componentes e sobre o sistema. Para Puente et al (2002), o FMEA permite que problemas potenciais sejam analisados, possíveis defeitos em produtos sejam identificados antes de chegarem ao cliente final, cujos efeitos no sistema completo podem ser estudados e decisões mais assertivas de controle podem ser tomadas, tanto para o FMEA de produto (DFMEA) quanto de processo (PFMEA).

Na elaboração do FMEA, cada componente é examinado de forma a identificar seus possíveis modos de falha. Para cada modo de falha são atribuídos três valores: a probabilidade de ocorrência de um modo de falha ("O"), a severidade do impacto de tal falha ("S") e a capacidade dos meios de detecção desta falha antes que ela realmente ocorra ("D"). A simples multiplicação destas três variáveis gera o valor do risco, denominado NPR (MADDOX, 2005).

Apesar do FMEA colaborar com a priorização dos efeitos mais críticos dos modos de falha em análise, possibilitando assim uma priorização dos recursos disponíveis, este requer uma análise de cada componente de um sistema, fato extremamente trabalhoso que pode rapidamente exceder os recursos disponíveis (TRAMMELL et al, 2004). De acordo com Puente et al. (2002) pode-se fazer as seguintes críticas ao FMEA:

- A avaliação oferecida pelo NPR nem sempre pode ser aferida pelos meios de detecção ("D");

- Não há uma regra algébrica precisa para a determinação dos índices ocorrência (“O”) e detecção (“D”);

- O cálculo do NPR baseado no produto dos três índices também pode causar distorções, pois enquanto a probabilidade de não-detecção e sua respectiva pontuação seguem uma função linear, a relação entre a probabilidade de ocorrência de uma falha e sua pontuação não necessariamente é linear;

- Diferentes pontuações de "O" e "D" podem levar a um mesmo NPR, apesar do risco envolvido ser completamente diferente;

- O NPR não consegue mensurar e efetividade das ações de melhoria propostas.

Ainda como uma crítica ao FMEA, para as fases de desenvolvimento de novos produtos, além de prever possíveis falhas dos mesmos, é necessário gerir de uma forma mais ampla os riscos associados a tais falhas, como por exemplo, possíveis atrasos, desvios de escopo e orçamento, análises estas que a ferramenta não proporciona (SEGISMUNDO; MIGUEL, 2006). O FMEA por si só não constitui um 
método de gerenciamento de riscos, devendo seus pontos fortes serem integrados à dimensões de análise como os impactos nos prazos, escopo, custos, ainda com uma tratativa temporal de evolução dos riscos (TRAMMELL et al, 2004). Para Pollock (2005) um dos maiores riscos incorridos pelos projetos que atualmente utilizam FMEA é que os times envolvidos normalmente terminam seu trabalho numa determinada fase dos mesmos e mudam-se imediatamente para a próxima, abandonando totalmente ou delegando para a área funcional de qualidade da empresa o acompanhamento das ações do FMEA.

Alguns poucos autores recentemente desenvolveram trabalhos propondo uma visão mais integrada da gestão dos riscos técnicos e seus impactos (PUENTE et al., 2002; TRAMMELL et al., 2004; CARBONE et al., 2004).Para Trammell et al (2004), em sua proposição de um modelo integrado de análise de risco para projetos, o FMEA, utilizado de maneira integrada, apresenta várias vantagens, como especificações de clientes podem ser identificadas; prazos e custos de lançamento de novos produtos podem ser reduzidos (como re-projetos e modificações são evitadas e muitos testes podem ser eliminados), aumento da qualidade e confiabilidade do produto e processo, o que leva a uma maior segurança e responsabilidade no processo de manufatura além do aumento da satisfação do cliente. Seu modelo baseia-se em um fluxo de processo de gerenciamento de riscos que vai desde a identificação e priorização dos sistemas relevantes para a análise até o acompanhamento do risco (NPR) ao longo do tempo e em função da tolerância ao risco do tomador de decisão. Puente (2002), por sua vez, propôs a adoção de um sistema de suporte à decisão baseado nos princípios do FMEA, o qual possibilita uma priorização das ações não apenas de forma decrescente com o NPR, mas sim, usando uma escala de prioridades definida pelo tomador de decisão. Carbone e Tippet (2004), propôs uma extensão do FMEA tradicional para a aplicação também nas dimensões custo e prazo de projetos, tornando-o assim, uma ferramenta mais abrangente de gestão de riscos. Ė neste sentido que se acredita que uma visão mais integrada do processo de gerenciamento de riscos e seus sub-processos é de suma importância para o sucesso de um projeto (SHENHAR et al., 2002, CARBONE et al., 2004).

Para o PMBOK, em sua última versão (PMI, 2004), a gestão de risco de um projeto inclui os processos que tratam da identificação, análise, respostas, monitoramento, controle e planejamento do gerenciamento de riscos. Os objetivos do gerenciamento de riscos do projeto são aumentar a probabilidade e o impacto dos eventos positivos e diminuí-los para eventos adversos ao projeto (PMI, 2004), orientando assim, a alocação de recursos de engenharia e a tomada de decisão ao longo do processo de desenvolvimento (PATÉ-CORNELL, 2002).

Os processos de gerenciamento de riscos para o PMI (2004) são:

- Planejamento do Gerenciamento de Riscos: decisão de como abordar, planejar e executar as atividades as atividades de gerenciamento de riscos de um projeto;

- Identificação dos riscos: determinação dos riscos que podem afetar o projeto e documentação de suas características;

- Análise qualitativa dos riscos: priorização dos riscos para análise ou ação adicional subseqüente através da avaliação e combinação de sua probabilidade de ocorrência e impacto;

- Análise quantitativa dos riscos: análise numérica dos efeitos dos riscos identificados nos objetivos gerais do projeto;

- Planejamento de respostas a riscos: desenvolvimento de opções de ações para aumentar as oportunidades e reduzir as ameaças aos objetivos do projeto;

- Monitoramento e controle dos riscos: acompanhamento dos riscos identificados, monitoramento dos riscos residuais, identificação dos novos riscos, execução dos planos de respostas aos riscos e avaliação de sua eficácia durante todo o ciclo de vida do projeto. 
Carbone e Tippet (2004), no entanto, declaram que muitos autores da área e o próprio PMBOK cometem algumas discrepâncias em suas definições, as quais geram confusão. Por exemplo, na discussão sobre ferramentas e técnicas de análise qualitativa do risco, o PMBOK (2004) mistura a aplicação das palavras "impacto" e "conseqüências" do risco. O NPR também pode ser definido como Risk Score (PMI, 2004), Severidade do Risco (Graves, 2000), o que pode ser facilmente confundido com o conceito do índice severidade ("D") para se estimar o risco no FMEA.

A Tabela 1 apresenta um exemplo de impactos negativos de definições que podem ser utilizadas na avaliação dos impactos de risco relacionados aos quatro principais objetos do projeto: custo, qualidade, prazo e escopo. São ilustradas na tabela tanto a abordagem relativa quanto numérica (neste caso, não linear), porém não necessariamente estes números estão diretamente ligados em termos de equivalência.

Tabela 1 - Definição de escala de impacto para as quatro dimensões de um projeto - adaptada de PMI (2004).

\begin{tabular}{|l|c|c|c|c|c|}
\hline \multicolumn{6}{|c|}{ Condições definidas para escalas de impacto de um risco em objetivos importantes do projeto } \\
\hline $\begin{array}{l}\text { Objetivo } \\
\text { do projeto }\end{array}$ & $\begin{array}{c}\text { Muito baixo / } \\
0,05\end{array}$ & Baixo / 0,10 & $\begin{array}{c}\text { Moderado / } \\
0,20\end{array}$ & Alto / 0,40 & $\begin{array}{c}\text { Muito alto / } \\
0,80\end{array}$ \\
\hline Custo & $\begin{array}{c}\text { Aumento de } \\
\text { custo não } \\
\text { significativo }\end{array}$ & $\begin{array}{c}\text { Aumento de } \\
\text { custo }<10 \%\end{array}$ & $\begin{array}{c}\text { Aumento de } \\
\text { custo de } 10 \text { a } \\
20 \%\end{array}$ & $\begin{array}{c}\text { Aumento de } \\
\text { custo de } 20 \text { a } \\
40 \%\end{array}$ & $\begin{array}{c}\text { Aumento de } \\
\text { custo }>40 \%\end{array}$ \\
\hline Tempo & $\begin{array}{c}\text { Aumento de } \\
\text { tempo não } \\
\text { significativo }\end{array}$ & $\begin{array}{c}\text { Aumento de } \\
\text { tempo }<5 \%\end{array}$ & $\begin{array}{c}\text { Aumento de } \\
\text { tempo de } 5 \text { a } \\
10 \%\end{array}$ & $\begin{array}{c}\text { Aumento de } \\
\text { tempo de } 10 \text { a } \\
20 \%\end{array}$ & $\begin{array}{c}\text { Aumento de } \\
\text { tempo }>20 \%\end{array}$ \\
\hline Escopo & $\begin{array}{c}\text { Diminuição do } \\
\text { escopo quase } \\
\text { imperceptível }\end{array}$ & $\begin{array}{c}\text { Áreas menos } \\
\text { importantes do } \\
\text { escopo afetadas }\end{array}$ & $\begin{array}{c}\text { Áreas } \\
\text { importantes do } \\
\text { escopo afetadas }\end{array}$ & $\begin{array}{c}\text { Redução } \\
\text { inaceitável para } \\
\text { o patrocinador }\end{array}$ & $\begin{array}{c}\text { Item final do } \\
\text { projeto sem } \\
\text { nenhuma } \\
\text { utilidade }\end{array}$ \\
\cline { 1 - 4 } Qualidade & $\begin{array}{c}\text { Degradação da } \\
\text { qualidade quase } \\
\text { imperceptível }\end{array}$ & $\begin{array}{c}\text { Somente } \\
\text { aplicações mais } \\
\text { críticas afetadas }\end{array}$ & $\begin{array}{c}\text { Redução exige } \\
\text { a aprovação do } \\
\text { patrocinador }\end{array}$ & $\begin{array}{c}\text { Redução } \\
\text { inaceitável para } \\
\text { o patrocinador }\end{array}$ & \\
\hline
\end{tabular}

São através destas definições e limitações que a área de gestão de riscos apresenta, que se gerou a motivação para o desenvolvimento da presente pesquisa, na tentativa de colaborar, mesmo que até o momento parcial e modestamente, para o desenvolvimento da mesma. Os métodos utilizados na pesquisa são descritos na seção 3 .

\section{Métodos de pesquisa}

A presente pesquisa possui como objetivo geral analisar o papel do FMEA no processo de tomada de decisão em desenvolvimento de produtos em uma empresa automobilística. A opção metodológica usada foi a de estudo de caso, cujos critérios de seleção foram além do setor da empresa, a importância da área de gestão de riscos de desenvolvimento de novos produtos. Para se realizar o estudo na organização, optou-se pelo levantamento de dados documentais de projetos realizados nos últimos quatro anos pela empresa (Yin, 1994), visto que foi neste período que se realizou seu último grande programa estratégico (denominado Programa Alfa) antes do atual (Programa Beta), o qual é o piloto do estudo.

Os dados, do tipo primário, foram coletados através de análise de documentos, base de dados e relatórios técnicos do Programa Alfa, como cronogramas, relatórios de resultados de simulações, testes de bancada e veiculares, e analisados de forma comparativa com os primeiros dados disponíveis do Programa Beta, o qual ainda encontra-se em suas fases iniciais do ciclo de vida. Como variáveischave para a análise comparativa dos programas, utilizou-se até o momento: número de protótipos 
veiculares construídos, número de iterações (ou loopings) de projeto e planejamento de teste e número de falhas no período de garantia do Programa Alfa.

A proposição do trabalho consiste na sistematização e utilização da gestão de riscos técnicos através do uso da ferramenta FMEA para otimizar o processo de tomada de decisão no ambiente de desenvolvimento de novos produtos da empresa objeto de estudo, gerando-se inferências que colaborem com pesquisas e estudos subseqüentes na área. Além da proposição base e da necessidade crescente de gestão de riscos técnicos já mencionadas nos tópicos anteriores, outras necessidades específicas identificadas pela empresa objeto de estudo motivaram o desenvolvimento da presente pesquisa, como por exemplo:

- Análise mais detalhada e individual de cada possível modo de falha. Tal análise possibilitaria uma visão mais clara do "peso" de cada modo de falha, bem como, proporcionaria um acompanhamento mais detalhado das ações ao componente;

- Necessidade de se iniciar a identificação dos riscos desde as fases de conceituação e projeto, iniciando-se assim toda a cadeia mais cedo e podendo-se tomar ações mais eficazes para minimizar o impacto;

- Composição de um risco para o veículo completo, e não somente para seus sistemas e componentes, de forma a avaliar o risco para o cliente final interno - Gerente de Projeto - e externo;

Com base nos critérios apontados, foi selecionada uma empresa do setor automobilístico nacional de origem alemã e sediada no Brasil há 50 anos, atuando no setor de caminhões (leves urbanos a extrapesados fora-de-estrada) e ônibus (plataformas urbanas e rodoviárias) para os mercados do Brasil, América Latina, África do Sul e Oriente Médio e, cujos resultados do levantamento de campo serão apresentados nas seções 5 e 6 deste artigo. A empresa utiliza o FMEA para novos projetos de produto desde 1989, porém de forma sistêmica e integrada aos conceitos de gestão de riscos, apenas à partir de 2005, tendo o Programa Beta como piloto. Os conceitos utilizados serão apresentados na seção 4. Sua gestão de projetos está baseada nos conceitos e áreas de conhecimento propostos pelo PMBOK (PMI, 2004), apresentando uma metodologia própria em gestão de projetos, porém estando ainda no nível dois (básico) de maturidade proposto pelo PMMM (Project Management Maturity Model)

(CARVALHO; SEGISMUNDO, 2006).

\section{Conceito de gerenciamento de riscos técnicos utilizando FMEA em uma montadora de veículos}

A partir do exposto anteriormente, a empresa adotou o conceito exposto na Figura 1 para o seu programa estratégico mais recente (Programa Beta). Basicamente, adotou-se um DFMEA estruturado para um veículo completo genérico. Tal DFMEA, denominado Master DFMEA, possui três níveis de detalhamento: sistemas, subsistemas de primeiro nível e de segundo nível. São detalhados para cada um deles a sua função primária, seus modos de falha principais e secundários, além de quais legislações o mesmo está sujeito a atender (caso necessário). Ainda, podem ser assinaladas as características especiais relativas à segurança ou legislação que o projeto do mesmo deve levar em conta. Ao se conhecer o escopo de um novo projeto "A", por exemplo, pode-se consultar o Master DFMEA e se decidir quais e quantos DFMEAs são necessários para este novo projeto. 
Master DFMEA - Veículo

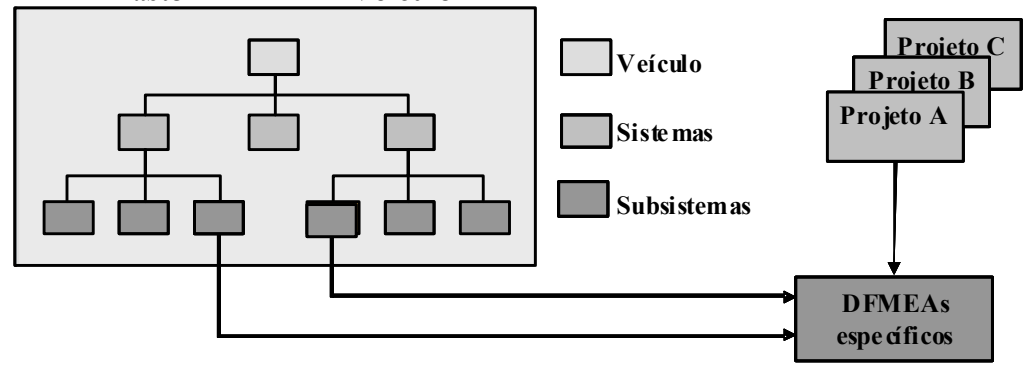

Figura 1 - Master DFMEA e sua correlação com projetos específicos.

Como principais características e vantagens do sistema, pode-se citar:

- Geração automática de uma lista de todas as características especiais relativas à segurança de um dado projeto;

- Base sólida para auxílio à tomada de decisão de quais e quantos sistemas e subsistemas de um projeto necessitam de DFMEAs, otimizando assim, recursos e tempo (MADDOX, 2005);

- Possibilidade de uma visão integrada novo projeto, mesmo em suas fases iniciais, direcionando os esforços e recursos (MADDOX, 2005);

Assim, com o intuito de sistematizar quais DFMEAs são necessários para um dado projeto, junto à necessidade de se quantificar e monitorar os riscos durante o andamento deste mesmo projeto, a empresa objeto de estudo percebeu a necessidade de se integrar estes conceitos. O macro-diagrama do processo de gerenciamento integrado de riscos técnicos da área de desenvolvimento de produtos pode ser visualizado no diagrama de processo da Figura 2.

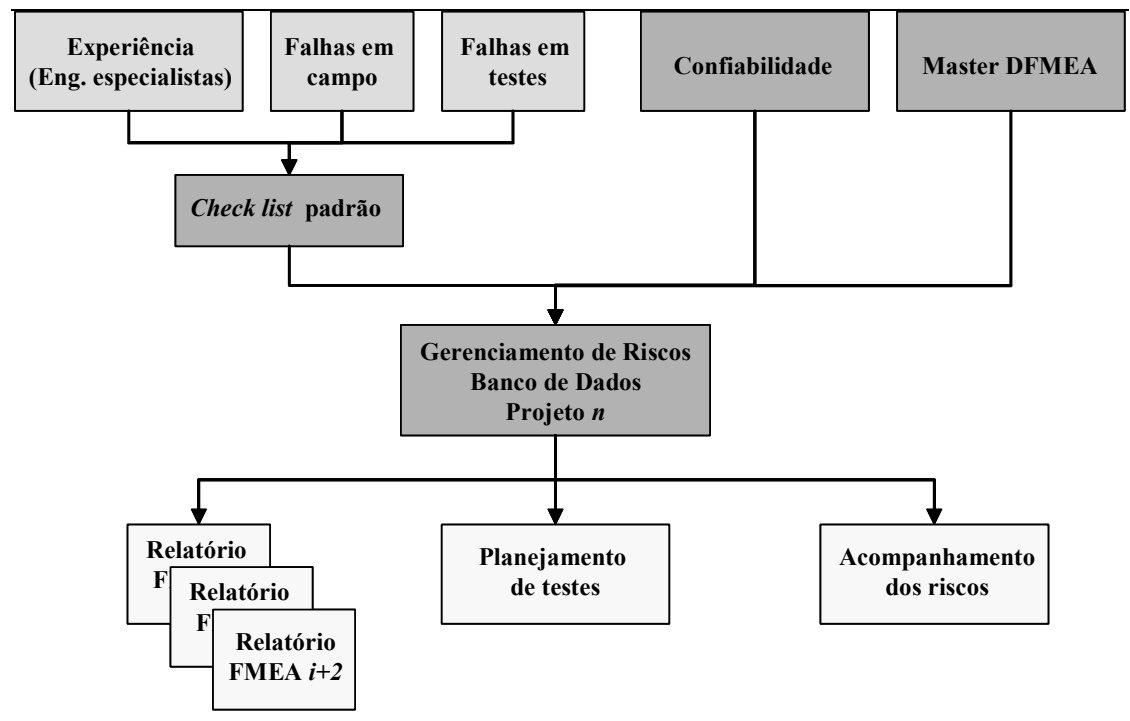

Figura 2 - Macro-diagrama do sistema de gestão de riscos.

Como principais entradas (parte superior do diagrama mostrado na Figura 2), têm-se a experiência dos engenheiros especialistas e as informações advindas de falhas em testes e falhas no campo (garantia) 
de produtos similares já em produção seriada. Ao se realizar um DFMEA, estas três entradas ocorrem por meio de um check list padrão, o qual através de equações que serão descritas mais a frente, quantifica os valores de probabilidade de ocorrência de um possível modo de falha ("O”), o impacto para o cliente caso tal falha ocorra ("S") e a capacidade dos mecanismos de testes e simulação de prever uma possível falha ainda nas fases de desenvolvimento ("D"). Outras entradas importantes são as informações já contidas e acumuladas gradativamente no Master DFMEA sobre o sistema ou componente em questão e as metas de confiabilidade especificadas no projeto em questão.

Como principais saídas, tem-se o próprio Master DFMEA, DFMEAs específicos de projetos, relatórios gerenciais de acompanhamento da evolução do risco ao longo do projeto, sempre de forma comparativa entre planejado e realizado, e o planejamento de testes.

O macro-diagrama exposto na Figura 2 pode ser detalhado através do fluxo completo de gestão de riscos técnicos utilizando FMEA, conforme apresentado pela Figura 3. Após determinação dos sistemas, subsistemas ou componentes que são pertinentes da realização de um DFMEA Figura 1, inicia-se o DFMEA propriamente dito, com a determinação dos possíveis modos e efeitos de falhas e suas causas. A determinação dos valores $\mathrm{O}, \mathrm{S}$ e $\mathrm{D}$ é realizada pelo grupo de engenheiros durante $\mathrm{o}$ processo do DFMEA, com o auxílio de um check list padrão, gerando um NPR para cada causa de cada modo de falha. O NPR é dividido em três faixas: verde (risco aceitável, nenhuma ação é necessária); amarelo (risco moderado, pelo menos uma ação é necessária) e vermelho (risco alto, uma ou mais ações são necessárias), procurando assim otimizar críticas clássicas a sua determinação (PUENTE, 2002). Desta maneira, todos os riscos amarelos ou vermelhos têm suas ações de melhoria monitoradas periódica e sistematicamente num ciclo PDCA (Plan, Do, Check and Act) até se encontrarem no nível aceitável e o sistema ser considerado aprovado para o processo de industrialização (manufatura e montagem).

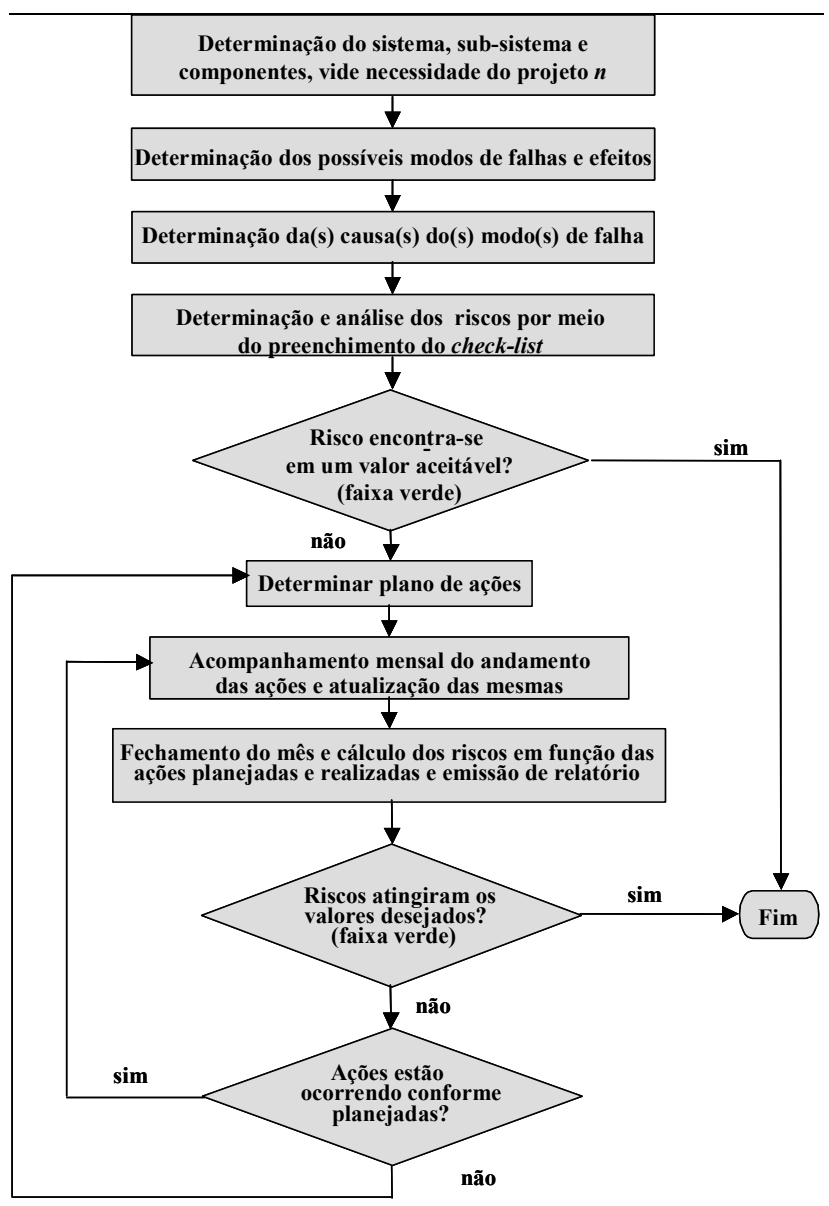


Figura 3 - Fluxograma detalhado do processo de gerenciamento de riscos técnicos de projetos da empresa objeto de estudo.

O risco é calculado da seguinte maneira (como num DFMEA convencional):

R ou NPR(i) $=\mathrm{O}(\mathrm{i}) \times \mathrm{S}(\mathrm{i}) \times \mathrm{D}(\mathrm{i})$

onde:

$\mathrm{R}(\mathrm{i})=$ Risco médio do sistema, subsistema ou componente i;

$\mathrm{O}(\mathrm{i})=$ Ocorrência, ou probabilidade dos possíveis modos de falha do sistema, subsistema ou componente i;

$\mathrm{S}(\mathrm{i})=$ Impacto ou severidade percebida pelo cliente no subsistema ou componente $\mathrm{i}$, caso um modo de falha previsto ocorra;

D(i) = Detecção, ou quão eficaz são os testes ou simulações ao prever um modo de falha.

As três variáveis acima citadas (Ocorrência, Severidade e Detecção) são definidas por meio da composição de notas que o sistema atribui a perguntas que os engenheiros respondem através do check list padrão. O cálculo baseia-se em uma média ponderada dos fatores, com relação a pesos que o administrador do sistema pode atribuir para cada ação mitigadora de riscos (reprojeto, testes de bancada, testes veiculares, etc.), como no exemplo abaixo (valores fíctícios):

Possíveis ações para se diminuir o risco através da variável "Detecção" (variação possível entre 10 péssima - e 0 - ótima)

- Pré-teste em bancada (fator $n$ ) $8 \times n$

- Sem nenhum teste em veículo (fator $2 \mathrm{n}$ ) $10 \times 2 \mathrm{n}$

- Teste de durabilidade em veículo (fator 4n) 4 x 4n

Assim, tem-se:

$D(i)=(8 \times n+10 \times 2 n+4 \times 4 n) /(n+2 n+4 n)$

Para o cálculo do risco do veículo como um todo, tem-se um fator de qualidade (FQ), o qual representa a importância de cada sistema, subsistema e componente para a funcionalidade básica do caminhão para o cliente. O cálculo é realizado através de média ponderada do risco de cada sistema, subsistema e componente pelo fator FQ, de forma similar ao exemplo dado sobre "Detecção", conforme detalhado em Segismundo e Miguel (2006).

5 Tomada de decisão no desenvolvimento de produto de uma montadora de veículos com base no gerenciamento de riscos - primeiros resultados

Na empresa objeto de estudo, o padrão corporativo de gestão de riscos técnicos no ambiente de desenvolvimento de produto é baseado na utilização de FMEA, conforme apresentado na seção 4. Para tal, a classificação dos riscos (NPR) apresenta três tipos de respostas em função da graduação do mesmo, conforme ilustra a Figura 4. O risco pode ser aceitável, entre 1 a 64 (faixa verde, ou na Figura 4 representado por cinza claro), médio entre 65 a 343 (faixa amarela, ou cinza escuro na Figura 4), e alto entre 344 e 1000 (faixa vermelha, ou preto na Figura 4). Estas faixas de riscos são obtidas através da multiplicação das variáveis $\mathrm{O}, \mathrm{S}$ e $\mathrm{D}$, as quais variam de 1 a 10, como num FMEA convencional. 
Para a faixa verde de riscos, por exemplo, os valores permitidos para $\mathrm{O}, \mathrm{S}$ e $\mathrm{D}$ variam entre 1 e $4 \mathrm{e}$, portanto, esta faixa tem seu valor mínimo igual a 1 (que tende ao vértice da reapresentação cartesiana das variáreis da Figura 4) e seu valor máximo igual a 64 (plano onde as três variáveis assumem valor ou classe 4$)$.

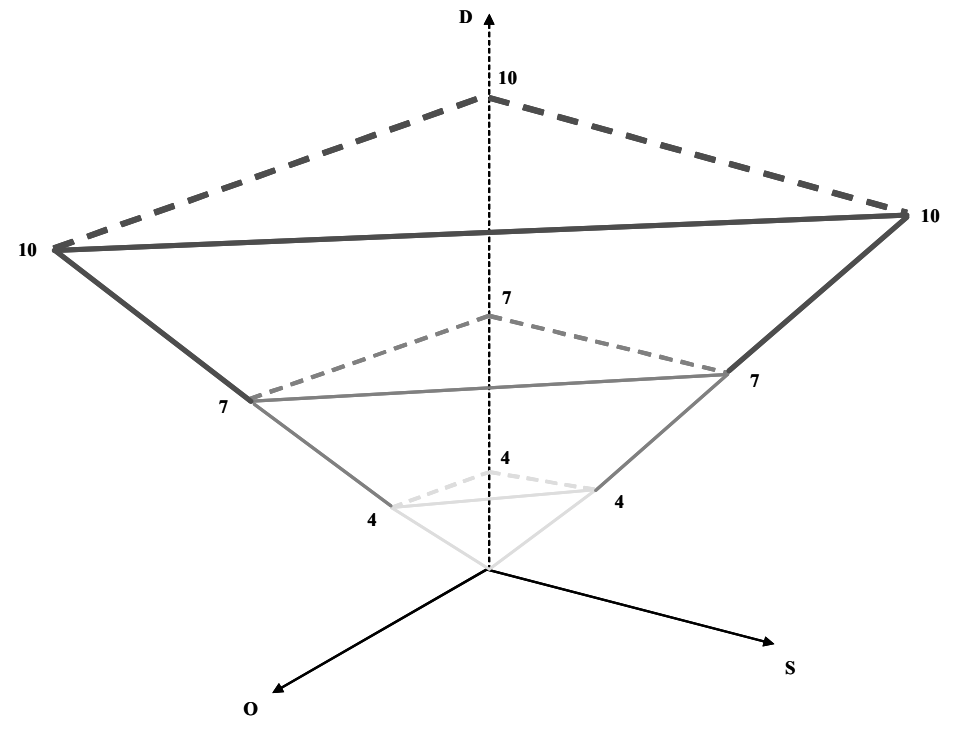

Figura 4 - Graduação do Risco (NPR).

Para cada plano de ação gerado por um DFMEA, necessários quando se tem um risco médio ou alto, é associada uma função que pode ser linearmente decrescente, de decréscimo imediato no início ou de decréscimo apenas na conclusão da mesma, indicando como o risco (NPR) deverá se comportar à medida que a ação evoluir conforme seu plano. Em uma distribuição linear, por exemplo o acúmulo de horas, ciclos ou distância percorrida por um protótipo em seus testes de simulação estrutural, o risco cai gradualmente com relação ao valor planejado à medida que o plano de ação que se utiliza destes recursos evolui. Outras ações podem ocasionar queda no NPR apenas após completadas com sucesso, como por exemplo, quando do recebimento do resultado de um cálculo estrutural. A Figura 5 exibe as três opções de distribuição permitidas pelo sistema de gerenciamento de riscos.

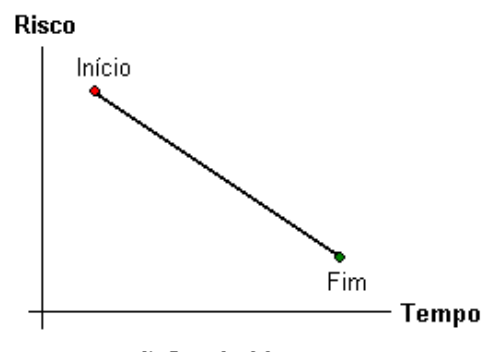

1) Queda Linear

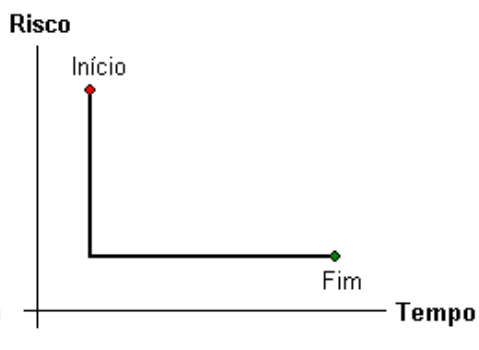

2) Queda Abrupta no Início

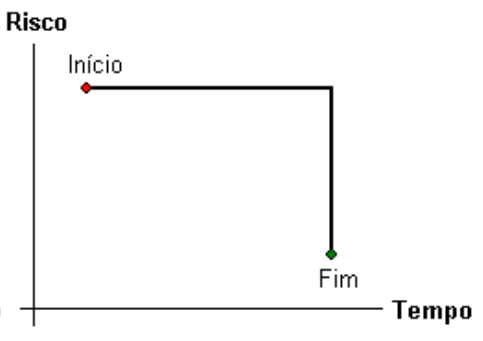

3) Queda Abrupta na Conclusão

Figura 5 - Funções de evolução do risco com o andamento positivo dos planos de ação.

Uma vez realizados todos os DFMEAs necessários a um projeto, com suas respectivas ações para a redução do risco técnico, inicia-se a fase de acompanhamento das mesmas. Para tal, pode-se gerar gráficos ou suas respectivas listagens, como os exibidos nas Figuras 6 a 8.

A Figura 6 exibe o controle dos riscos associados a um único componente do projeto ao longo tempo, neste caso do sub-sistema "Suportes do Tanque", relativo ao sistema "Tanque de Combustível". Podese observar a evolução planejada do risco, representada pela linha que decresce conforme a 
composição das três possíveis funções de redução dos mesmos, associadas às diferentes ações tomadas ao longo do tempo para se atingir o nível de risco aceitável (NPR máximo igual a 64, vide faixa cinza entre NPR 0 a 64 da Figura 6). As barras representam a evolução real do risco (NPR) para o subsistema "Suportes do Tanque". As barras em cinza claro apresentam que a queda do risco ocorreu conforme o previsto para o mês $i$, enquanto as barras cinza escuro representam que o risco não evoluiu conforme o esperado (como no mês de Junho na Figura 6), ou seja, tem-se um atraso ou um resultado negativo de uma das ações planejadas.

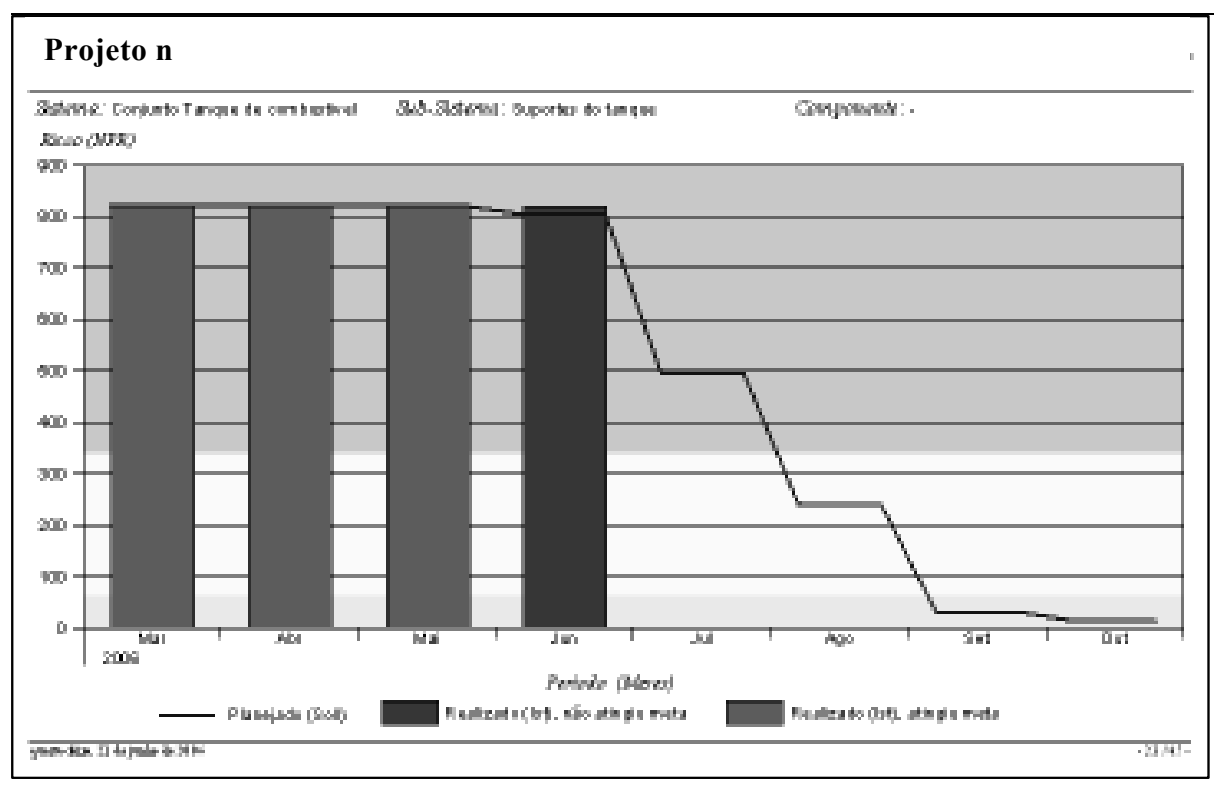

Figura 6 - Evolução do risco para um componente - planejado versus realizado.

A Figura 7 exibe o risco total médio do projeto n, da mesma maneira que a exposta na Figura 6 para um dado sub-sistema. Neste caso, o projeto $n$ representado trata-se do Programa Beta, cuja evolução do NPR é composta da média de todos os NPRs de seus sistemas e subsistemas, ponderada pelo fator FQ (vide seção 4 (2)). Pode-se observar que o NPR do Programa Beta era cerca de 230 em Março e, caso todas os planos de ação (sou simplesmente "ações") planejadas sejam executadas nos prazos e resultados previstos, o NPR evoluirá para 130 em Outubro, valor ainda acima do máximo normalmente aceitável (NPR igual a 64). Tal informação pode colaborar para o processo de decisão, onde a diretoria pode aceitar o planejamento mostrado na Figura 7, correndo seus riscos ou, por exemplo, re-priorizar recursos para que mais ações, ou ações mais dispendiosas, sejam tomadas para se atingir um NPR menor em Outubro. 


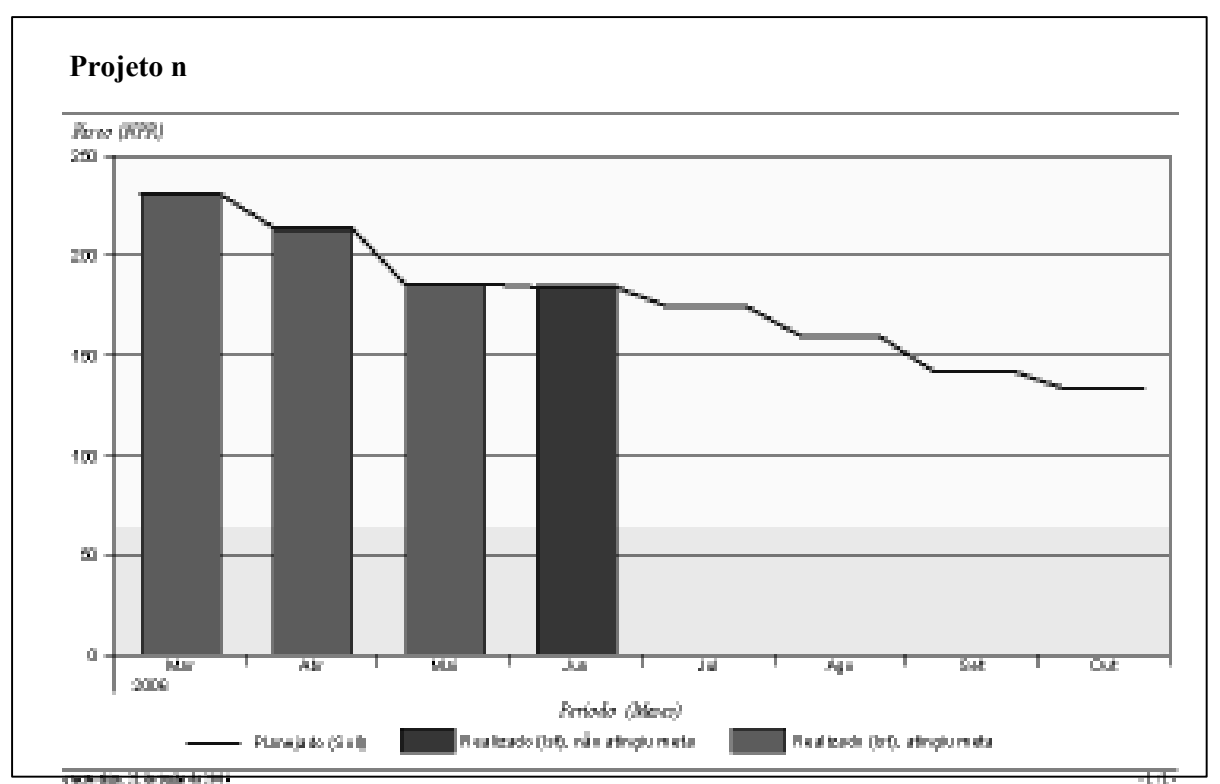

Figura 7 - Evolução de todos os riscos técnicos de um projeto ou programa - planejado versus realizado.

A Figura 8, por sua vez, exibe a quantidade total planejada e real de sistemas, sub-sistemas ou componentes por faixa de risco (NPR aceitável, médio e alta) e sua evolução com o tempo, para o Programa Beta. Por exemplo em Abril, num total de 45 sistemas, sub-sistemas ou componentes que compõe os projetos do Programa Beta, 8 se encontravam na faixa vermelha de risco, 27 na faixa amarela e 10 já na faixa verde. Caso todas as ações ocorram conforme plano, prevê-se que em Outubro ter-se-á 2,25 e 18 respectivamente.

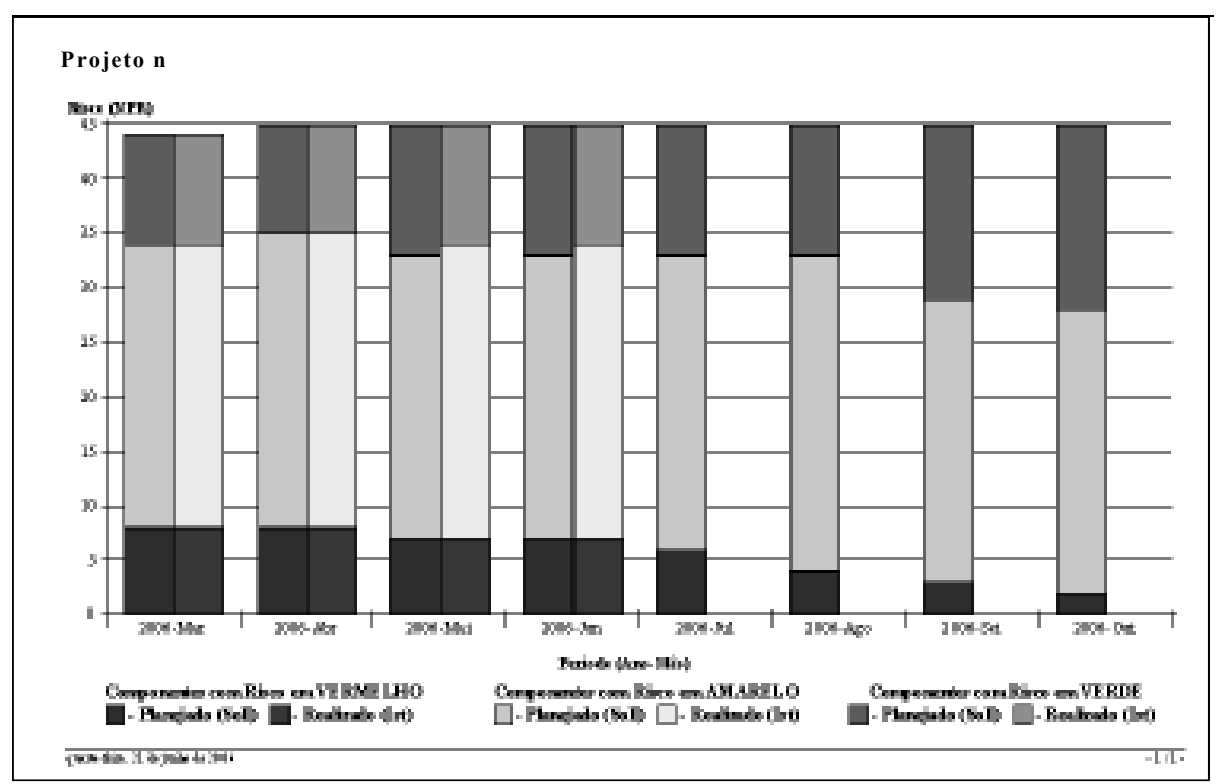

Figura 8 - Evolução da quantidade de componentes por faixa de risco - planejado versus realizado.

Tal acompanhamento proporciona ao corpo gerencial da diretoria de desenvolvimento de produtos uma visão geral do andamento dos riscos técnicos de seus projetos, podendo assim, tomar decisões mais rápidas e assertivas quanto a alocação de seus recursos humanos, financeiros e físicos, como 
compartilhamento de instalações de bancos de provas, equipamentos de medições, etc. (TRAMMELL et al, 2004).

\section{Outros resultados relevantes e próximos passos}

Como outros resultados parciais porém relevantes do estudo, pode-se citar que a utilização do conceito de gestão de riscos técnicos baseada em FMEA como parte fundamental no processo de tomada de decisão no desenvolvimento de novos produtos vêm sendo muito bem aceito pelos usuários (engenheiros e técnicos) e clientes internos tomadores de decisão (gerente de projeto, gerência funcional e diretoria da área de desenvolvimento de produtos). Tal fato fica evidenciado uma vez que todo novo projeto de produto à partir da implantação do conceito (SEGISMUNDO; MIGUEL, 2006) tem utilizado o mesmo e seus primeiros resultados têm sido alvo de discussão para tomada de decisão junto à alta diretoria.

O projeto piloto (Programa Beta) está na fase de inicial de seu ciclo de vida e pode-se citar que a utilização do conceito de gestão de risco com FMEA já durante esta fase, otimizou a necessidade de alguns loopings (cerca de 15\%) de projeto e planejamento de testes, focando em ações claramente direcionadas à minimização do risco da ocorrência de falhas que comprometessem a qualidade do produto ou seu prazo de aprovação, como por exemplo o aumento significativo do número de testes de bancada em lugar de alguns testes em veículo. A necessidade de protótipos para testes veiculares também foi reduzida em cerca de $35 \%$, devido às antecipações no planejamento de pré-testes que o gerenciamento de riscos propiciou. Com relação ao produto antecessor ao projeto piloto (Programa Alfa), das falhas ocorridas no período de garantia do mesmo (entre 2003 e 2005), apenas $24 \%$ passou por um processo de análise de risco, fato que evidencia a importância de tal processo e a necessidade de seu contínuo aprimoramento para a otimização do processo de tomada de decisão no desenvolvimento de novos produtos. Como conseqüências desta otimização, espera-se uma melhoria da qualidade dos produtos, custos de garantia e robustez do processo de desenvolvimento, a serem levantadas de forma quantitativa como próximos passos do presente estudo.

Tais próximos passos prevêem uma comparação dos resultados obtidos do projeto piloto com os de um projeto similar que não utilizou o conceito de gestão de riscos técnicos com FMEA, gerando assim, resultados que validem, ou não, a proposição de maneira mais robusta.

\section{Conclusões}

Como considerações finais, pode-se dizer que o presente estudo apresenta uma colaboração com o campo de pesquisa de gerenciamento de riscos técnicos em projetos, uma vez que analisa o papel de uma das técnicas mais difundidas de análise de risco técnico, o FMEA, num contexto mais amplo de gestão dos riscos como apoio à tomada de decisão ao longo da cadeia de desenvolvimento de novos produtos. Neste sentido, pode-se concluir que a pesquisa atingiu sua proposição de sistematizar e utilizar o FMEA na gestão de riscos técnicos num nível que colaborasse com o processo decisório e seus principais indicadores, apesar das limitações geradas pela necessidade de dados para uma maior validação no âmbito proposto da indústria automobilística. Para tal, é necessário tempo para que os primeiros resultados dos projetos pilotos (Programa Beta) sejam representativos em número, fato que deve ser explorado em estudo subseqüente.

Apesar de tais limitações, os primeiros resultados obtidos e citados nas seções 5 e 6 , mostram um avanço na sistematização e disponibilidade de algumas informações antes não disponíveis para o processo decisório, como a evolução temporal e comparativa entre o planejado e realizado do NPR de cada sistema, sub-sistema e componente, do NPR médio do projeto e do número de sistemas, subsistemas e componentes por faixa de risco. Além disso, ao se comparar os Programas Alfa e Beta, observou-se nas primeiras fases deste último uma ligeira otimização dos loopings de desenvolvimento e número de protótipos necessários para a aprovação dos componentes devido à utilização do conceito utilizado. Tais constatações levam a crer que, guardadas as suas limitações, possivelmente houve uma influência positiva no processo de tomada de decisão do desenvolvimento de forma a otimizar a alocação de seus recursos até o momento para o Programa Beta. 
Como potencial para trabalho futuro, tem-se a possibilidade de validação, através de métodos estatísticos aplicados a mais variáveis-chave de uma provável otimização do processo decisório em desenvolvimento de produtos, que a gestão de riscos técnicos pode vir a proporcionar e, ainda, propor num contexto mais amplo um conceito de gestão de riscos técnicos com a devida validação.

\section{Referências}

CARBONE, T.A.; TIPPETT, D.D. Project risk management using the Project risk FMEA. Engineering Management Journal, v.16, n.4, p. 28-35, 2004.

CARVAlHO, M.M.; RABECHINI Jr, R. Construindo Competências para Gerenciar Projetos: Teoria \& Casos. São Paulo: Atlas, 2005. 317p.

CARVALHO, M.M.; SEGISMUNDO, A. Maturidade em gestão de projetos: análise comparativa em 3 unidades de negócio do setor automobilístico. Artigo enviado ao XXVI ENCONTRO NACIONAL DE ENGENHARIA DE PRODUÇÃO; ENEGEP, Fortaleza, 2006.

DAVIES, T.J.C.; ARZYMANOW, A. The maturity of project management in different industries: An investigation into variations between project management models. International Journal of Project Management. v.21, p. 471-478, 2003.

GRAVES, R. Qualitative risk assessment. PM Network, v.14, n.10, p. 61-66, 2000.

HAIMES, Y.Y. Risk modeling, assessment and management. New York: Wiley, 1998.

KAPLAN, S. ; GARRICK, B.J. On the quantitative definition of risk. Risk Analysis, v.1, p. 11-27, 1981.

KAPLAN, S. The general theory of quantitative risk assessment. In: HAIMES, Y.Y., MOSER D.A. \& STAKHIV E.Z. RISK BASED DECISION MAKING IN WATER RESOURCES V, New York, 1991. American Society of Civil Engineers, p. 11-39, 1991.

KAPLAN, S. The words of risk analysis. Risk Analysis, v.17, p. 407-417, 1997.

KAPLAN, S.; HAIMES, Y.Y.; LAMBERT, J.H. Fitting hierarchical holographic modeling into the theory of scenario structuring and a resulting refinement to the quantitative definition of risk. Risk Analysis, v.21, n.5, p. 807-819, 2001.

KERZNER, H. Gestão de Projetos: as melhores práticas. Porto Alegre: Bookman, 2002. 519p.

KUMAR, R. L. Managing risks in IT projects: an options perspective. Information and Management, v.40, p. 63-74, 2002.

MADDOXX, M.E. Error apparent. Industrial Engineer, v.37, n.5, p. 40-44, 2005.

MARTINEZ, R.H. Processo de planejamento da resposta ao risco adotado por instituições financeiras no gerenciamento de risco em projetos de sistemas de informação: um estudo de casos múltiplos. Dissertação de Mestrado, USP/FEA. São Paulo, 2004.

PATÉ-CORNELL, E. Finding and fixing systems weaknesses: Probabilistic methods and applications of engineering risk analysis. Risk Analysis, v.22, n.2, p. 319-334, 2002. 
PEROBELLI, F. F. C. Um modelo para gerenciamento de riscos em instituições não financeiras: aplicação ao setor de distribuição de energia elétrica no Brasil. Tese de Doutorado, USP/FEA. São Paulo, 2004.

PMI, Project Management Institute. PMBOK - Project Management Body of Knowledge 2004. Minas Gerais: PMIMG, 2004.

POLLOCK, S. Create a simple framework to validate FMEA performance. ASQ Six Sigma Forum Magazine, v.4, n.4, p.27-34, 2005.

PUENTE, J.; PINO, R.; PRIORE, P.; FUENTE, D. A decision support system for applying failure mode and effects analysis. The International Journal of Quality \& Reliability Management, v.19, p. 137-150, 2002.

SEGISMUNDO, A.; MIGUEL, P.A.C. Gerenciamento de riscos em projetos: Proposição inicial de um conceito integrado para as fases de desenvolvimento de produto - Estudo em uma empresa automotiva. Artigo aceito para o XIV SIMPÓSIO INTERNACIONAL DE ENGENHARIA AUTOMOTIVA; SIMEA, São Paulo, 2006.

SHENHAR, A. J. One size does not fit all projects: exploring classical contingency domains. Management Science, v.47, n.3, p. 394-414, 2001.

SHENHAR, A. J.; RAZ, T.; DVIR, D. Risk management, project success, and technological uncertainty. $R \& D$ Management, v.32, n.2, p. 101-109, 2002.

SHIMIZU, T. Decisão nas organizações. 2. ed. São Paulo: Atlas, 2006. 419p.

SIMÕES, S.F. Aplicação de FMEA e FMECA na Tecnologia Submarina. In: CENPES/PDP/TS PETROBRAS, São Paulo, 2004.

TRAMMELL, S.R.; LORENZO, D.K.; DAVIS, B.J. Integrated hazard analysis: Using the strengths of multiple methods to maximize the effectiveness. Professional Safety, v.49, n.5, p. 29-37, 2004.

YIN, R. Case study research. New York: Sage Publications, 1994. 419p. 